\title{
Der Bewegungsförderer
}

GUNNAR GEUTER Nach seinem Studium entschied sich Physiotherapeut Gunnar Geuter dafür, die Behandlungsbank zu verlassen. Nun arbeitet er im öffentlichen Gesundheitsdienst. Ein nicht weniger bewegender Arbeitsplatz.

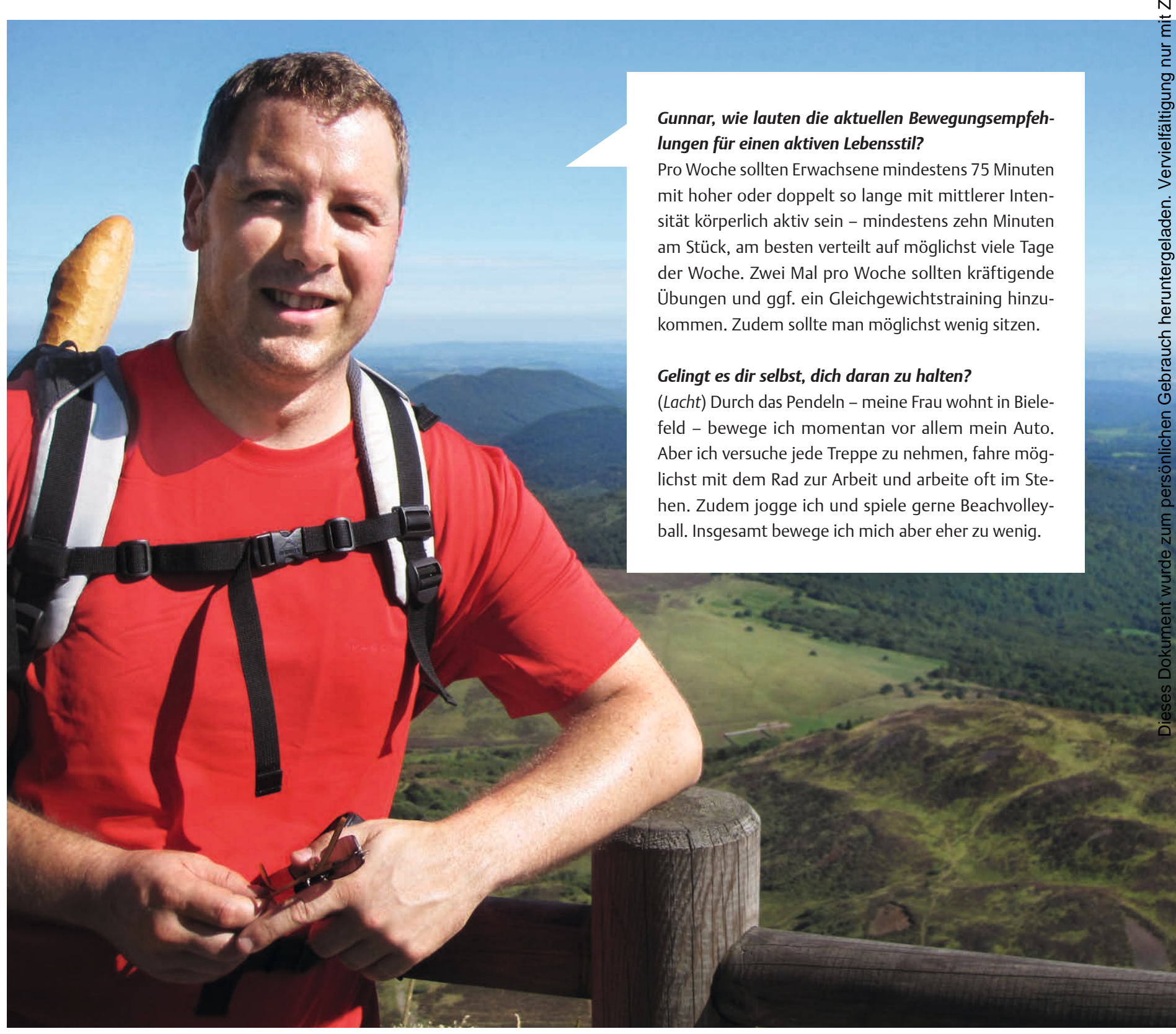




\section{Öffentlicher Gesundheitsdienst}

Gunnar Geuter ...

... ist Physiotherapeut, 35 Jahre alt und lebt in Bielefeld und Nürnberg. Er absolvierte den Diplom-Studiengang „Lehrer für Gesundheitsberufe“ an der FH Bielefeld und den Masterstudiengang Public Health an der Uni Bielefeld. Daneben war er schon Lehrer an einer Physiotherapieschule, Dozent in der beruflichen Weiterbildung und wissenschaftlicher Mitarbeiter an zwei Hochschulen.

2009 wurde Gunnar Geuter Dezernent am Landesinstitut für Gesundheit und Arbeit des Landes Nordrhein-Westfalen (LIGA.NRW). Dort baute er unter anderem das „Zentrum für Bewegungsförderung Nordrhein-Westfalen“ auf, das er auch fachlich leitete. Dessen Ziel ist, die Bewegung von Menschen im Altag zu fördern. Mittlerweile ist er nach Bayern zurückgekehrt. Als Leiter des Kommunalbüros für ärztliche Versorgung im Bayerischen Landesamt für Gesundheit und Lebensmittelsicherheit (LGL) berät er unter anderem Kommunen, welchen Beitrag sie leisten können, um die ärztliche Versorgung zu erhalten und zu verbessern.

Gunnar Geuter schätzt besonders, dass der Öffentliche Gesundheitsdienst (ÖGD) - im Gegensatz zu anderen Akteuren im Gesundheitssystem - interessenneutral und dem Gemeinwohl verpflichtet ist.

\section{Das Tätigkeitsfeld}

Der Öffentliche Gesundheitsdienst (ÖGD) schützt, verbessert und erhält die Gesundheit der Bevölkerung. Er unterstützt aber auch die gesundheitliche Versorgung Einzelner, etwa die von Menschen ohne einen gesicherten Aufenthaltsstatus. Einrichtungen des ÖGD gibt es auf der Ebene des Bundes (z. B. Robert-KochInstitut), der Länder (z. B. Gesundheitsministerien) und der Kommunen (z. B. Gesundheitsämter). Sie beobachten etwa die gesundheitliche Lage und Versorgung der Bevölkerung, wirken an Krankheitsprävention und -bekämpfung mit und überwachen Hygienevorschriften sowie Herstellung und Handel von Arzneimitteln und Medizinprodukten.

Neu für den ÖGD in Deutschland ist das von Gunnar Geuter aufgebaute und bis 2012 geleitete „Zentrum für Bewegungsförderung Nordrhein-Westfalen“. Es identifiziert, analysiert und kommuniziert evidenzbasierte Interventionen und Beispiele guter Praxis zur alltagsbezogenen Bewegungsförderung und unterstützt die Qualitätssicherung in diesem Feld. Darüber hinaus vernetzt das Zentrum die Akteure der Bewegungsförderung über Fachtagungen, Fachgespräche und Workshops. Zudem ermittelt es regionalspezifische Bedarfe der Bewegungsförderung und entwickelt bewegungsförderliche Strukturen weiter.

Zwei weitere Projekte von Gunnar Geuter waren ein Handbuch zu Bewegungsförderung und Gesundheit sowie ein Fachinformationsportal. Das Handbuch zeigt den aktuellen Forschungsstand zu den Zusammenhängen von Bewegungsförderung und Gesundheit und stellt unter anderem wirksame und qualitätsgesicherte Strategien der Bewegungsförderung vor. Es wendet sich an Leser aus Wissenschaft, Politik und Praxis. In dem Internetportal können Nutzer unter anderem evidenzbasierte Bewegungsempfehlungen für einen aktiven Lebensstil sowie qualitätsgesicherte Fachinformationen abrufen. Mit seinen Aktivitäten konnte Gunnar Geuter erfolgreich dazu beitra- gen, dass das Thema „Bewegungsförderung und Gesundheit" auf Landes- und auf kommunaler Ebene zunehmend berücksichtigt wird.

Gunnar Geuter leistet also einen Beitrag zur Gesunderhaltung auf Bevölkerungsebene.

\section{Zentrale Erkenntnisse}

$>$ Trotz Studien ist noch nicht genau geklärt, wie das Bewegungsverhalten der Bevölkerung langfristig gesteigert werden kann.

> Es gibt jedoch deutliche Hinweise dafür, dass eine Kombination aus Verhaltens- und Verhältnisprävention dafür nötig ist. Die meisten Programme zur Bewegungsförderung basieren auf rein verhaltensbezogenen Ansätzen und berücksichtigen zu selten Theorien zur Verhaltensänderung.

$>$ Bewegung ist auch dann gesundheitswirksam, wenn sie quasi „nebenbei“, also im Alltag, stattfindet.

\section{Fazit}

Zusammenfassend kann Gunnar Geuter festhalten, dass ...

$>$ Bewegungsförderung eine gesamtgesellschaftliche Aufgabe ist, die interdisziplinär, intersegmental und ressortübergreifend angegangen werden muss.

$>$ Lebenswelten bewegungsfreundlicher werden müssen: Verkehrsberuhigte Innenstädte regen z. B. dazu an, Erledigungen zu Fuß oder mit dem Fahrrad zu machen. Die gesunde Wahl sollte die einfachere Wahl sein.

> Städte und Gemeinden für genügend Bewegungs- und Freiflächen sorgen sollten.

$>$ Bewegungsförderung ein integraler Bestandteil von Physiotherapie sein sollte. Therapeuten sollten sich stärker mit Beratung und Begleitung bei Verhaltensänderungen befassen sowie wissenschaftliche Erkenntnisse und Theorien einbinden.

Eva Trompetter

$\Rightarrow$ Fachinformationsportal zum Thema
Bewegungsförderung: www.zfb.nrw.de 